\title{
Small-angle Thomson scattering of ultrafast laser pulses for bright, sub-100-fs x-ray radiation
}

\author{
Yuelin Li, Zhirong Huang, Michael D. Borland, and Stephen Milton \\ Advanced Photon Source, Argonne National Laboratory, Argonne, Illinois 60439
}

(Received 13 December 2001; published 5 April 2002)

\begin{abstract}
We propose a scheme for bright sub-100-fs x-ray radiation generation using small-angle Thomson scattering. Coupling high-brightness electron bunches with high-power ultrafast laser pulses, radiation with photon energies between 8 and $40 \mathrm{keV}$ can be generated with pulse duration comparable to that of the incoming laser pulse and with peak spectral brightness close to that of the third-generation synchrotron light sources of $\sim 10^{20}$ photons $\mathrm{s}^{-1} \mathrm{~mm}^{-2} \mathrm{mrad}^{-2}$ per $10^{-3}$ bandwidth. A preliminary dynamic calculation is performed to understand the property of this novel scattering scheme with relativistic laser intensities.
\end{abstract}

DOI: 10.1103/PhysRevSTAB.5.044701

PACS numbers: 52.38.Ph, 52.59.Px, 41.60.Ap, 41.75.Ht

\section{INTRODUCTION}

Thomson scattering of an intense laser beam from relativistic electron bunches is well known to generate frequency up-shifted photons in a narrow forward cone $[1,2]$. Recently, with the advent of high quality electron bunches generated with modern photoinjectors [3] and intense, ultrafast laser beams [4], a variety of Thomson scattering schemes have been used to generate bright, ultrafast x-ray pulses with duration as short as a few picoseconds, and sometimes even into the subpicosecond regime. For example, using the $90^{\circ}$ scattering scheme [5], $\mathrm{x}$-ray pulses as short as a few hundred femtoseconds have been generated [6,7]. With the head-on scattering scheme, bright picosecond $\mathrm{x}$-ray pulses have been achieved $[7,8]$, up to a peak photon flux of $10^{19}$ photons per second [8]. These x-ray pulses are essential tools for elucidating the ultrafast dynamics in solids and liquids in related chemical, biological, and physical sciences [9]. However, the pulse duration achieved so far, namely, subpicosecond durations also achieved by other means such as laser plasma radiation [10] and sliced synchrotron radiation [11], is still an order of magnitude too long for achieving the resolution at the atomic scale of a few femtoseconds $[10,12]$. It is possible to further shorten the pulse duration with these existing schemes, but only at the expense of severely decreased photon production or spectral brightness.

In this paper, we propose the use of the small-angle Thomson scattering (SATS) scheme as a unique solution to the conflicting interests of generating shorter pulse duration and higher spectral brightness. In this scheme, the $\mathrm{x}$-ray pulse duration is determined by that of the scattering laser pulse, in contrast to previous schemes where it is determined by the longitudinal or transverse electron bunch sizes. As such, SATS is capable of generating $\mathrm{x}$-ray radiation as fast as state-of-the-art ultrafast laser systems with peak spectral brightness close to that of third-generation synchrotron light sources, $20 \mathrm{fs}$ and $\sim 10^{20}$ photons s${ }^{-1} \mathrm{~mm}^{-2} \mathrm{mrad}^{-2}$ per $0.1 \%$ bandwidth in our example. To our knowledge, no such $\mathrm{x}$-ray source has been proposed or demonstrated except perhaps for the idea of manipulating the pulses from a future x-ray free-electron laser [13,14].

In Sec. II we give the analysis of the pulse duration, the spectral properties, and the spectral brightness of the SATS scheme in the linear Thomson scattering regime, while in Sec. III we present a preliminary dynamic calculation of the electrons when the laser intensity becomes relativistic. In Sec. IV we discuss the results, and in Sec. V we summarize the paper.

\section{SMALL-ANGLE THOMSON SCATTERING}

\section{A. Linear Thomson scattering}

Theoretically, Thomson scattering from a relativistic electron beam has been extensively studied both in the linear $[1,2]$ and the nonlinear regime $[15,16]$. In this section, for clarity, we adapt the linear theory, where the energy of the scattered photon is calculated using energy momentum conservation, and the scattering cross section is calculated using the theory of quantum electrodynamics [17]. With the scattering angle $\theta \ll 1$ and the relativistic factor of the electron bunch $\gamma \gg 1$, the energy of the scattered photon is

$$
E \approx E_{L} \frac{2 \gamma^{2}}{1+\gamma^{2} \theta^{2}}(1-\cos \phi),
$$

and the differential scattering cross section (after integration over the azimuthal angle) is

$$
\frac{d \Sigma}{d \theta} \approx 8 \pi r_{e}^{2} \gamma^{2} \frac{1+\gamma^{4} \theta^{4}}{\left(1+\gamma^{2} \theta^{2}\right)^{4}} \theta .
$$

Here $E_{L}$ is the energy of the incident photon, $\phi$ and $\theta$ are, respectively, the angles of the incident and scattered photons with respect to the electron propagation direction, and $r_{e}$ is the classical electron radius. When $\phi \ll 1$ at $\theta=0$, we have $\phi=\left(E / E_{L}\right)^{1 / 2} / \gamma$. 


\section{B. Pulse duration}

Note that from Eq. (1) an interesting scenario is created when one increases the electron beam energy while reducing the laser incidence angle to maintain the scattered photon energy constant. In this case the slippage between the incoming laser pulse and the scattered photons can be significantly reduced, thus limiting the longitudinal size of the emitter, favorable for short-pulse generation. Meanwhile, at higher electron energies, smaller divergence is expected for the scattered photons as a result of the Lorentz contraction effect.

Consider a Gaussian electron bunch with $\sigma_{x, y, z} \mathrm{rms}$ bunch sizes propagating along the $z$ axis and interacting with a Gaussian laser pulse propagating in the $x-z$ plane. The laser has an rms pulse duration of $c \tau_{L}<\sigma_{z}$, and the beams are matched in the vertical direction so that the laser beam waist $w_{0}=2 \sigma_{y}$. The pulse duration of the scattered photon burst is the convolution of three factors: the laser pulse length $\tau_{L}$, the lag between the scattered and the incident photons $(1-\cos \phi) t_{c} \approx \sigma_{x} \phi / 2 c$, and the projection of the transverse laser beam size along the $z$ axis, which is $(1-\cos \phi) t_{c}^{\prime}-\sigma_{y} \sin \phi / c \approx-\sigma_{y} \phi / 2 c$. Here $t_{c} \approx \sigma_{x} / c \sin \phi=\sigma_{x} / c \phi$ is the time needed for the laser pulse to cross the electron bunch (we assume $\sigma_{x} \geq \sigma_{y}$ ), and $t_{c}^{\prime} \approx \sigma_{y} \cos \phi / c \sin \phi$. Hence the pulse duration of the scattered photons is

$$
\tau \approx \tau_{L}\left[1+\left(1+\frac{\sigma_{x}^{2}+\sigma_{y}^{2}}{4 \tau_{L}^{2} c^{2}}\right) \phi^{2}\right]^{1 / 2} .
$$

Here $w_{0}=2 \sigma_{y}$ is used. Equation (3) reveals an important characteristic of the small-angle Thomson scattering scheme, i.e., its capability to generate an x-ray burst with pulse duration comparable to that of the incident laser. This occurs with reasonably focused electron bunches and for small enough laser incidence angle. Under this condition, the laser and electron beam sizes play no role in determining the pulse duration, and the short pulse length of the scattered radiation is the result of a "sliced" Thomson scattering in which only those electrons underlying the laser pulse are scattering the laser photons.

The result is depicted in Fig. 1(a), where the numerically calculated $\mathrm{x}$-ray pulse duration generated by scattering a FWHM 20-fs (rms duration of 8.5 fs) laser pulse off a 0.2-ps electron bunch is plotted as a function of the electron bunch transverse sizes and energies. The calculation is performed by numerically integrating the distribution function (assumed to be Gaussian) of the electron bunch and the laser pulse coupled by Eqs. (1) and (2). The laser wavelength is at $800 \mathrm{~nm}(1.55 \mathrm{eV})$, and the peak of the angle-integrated $\mathrm{x}$-ray spectra is kept at $8 \mathrm{keV}$ by adjusting the laser incidence angle over the range from 380 to $19 \mathrm{mrad}$. Clearly, x-ray pulse duration down to 20 to $30 \mathrm{fs}$ can be achieved, a regime so far available only at visible to vacuum ultraviolet wavelengths. In the calculation, a conservative normalized electron beam emittance
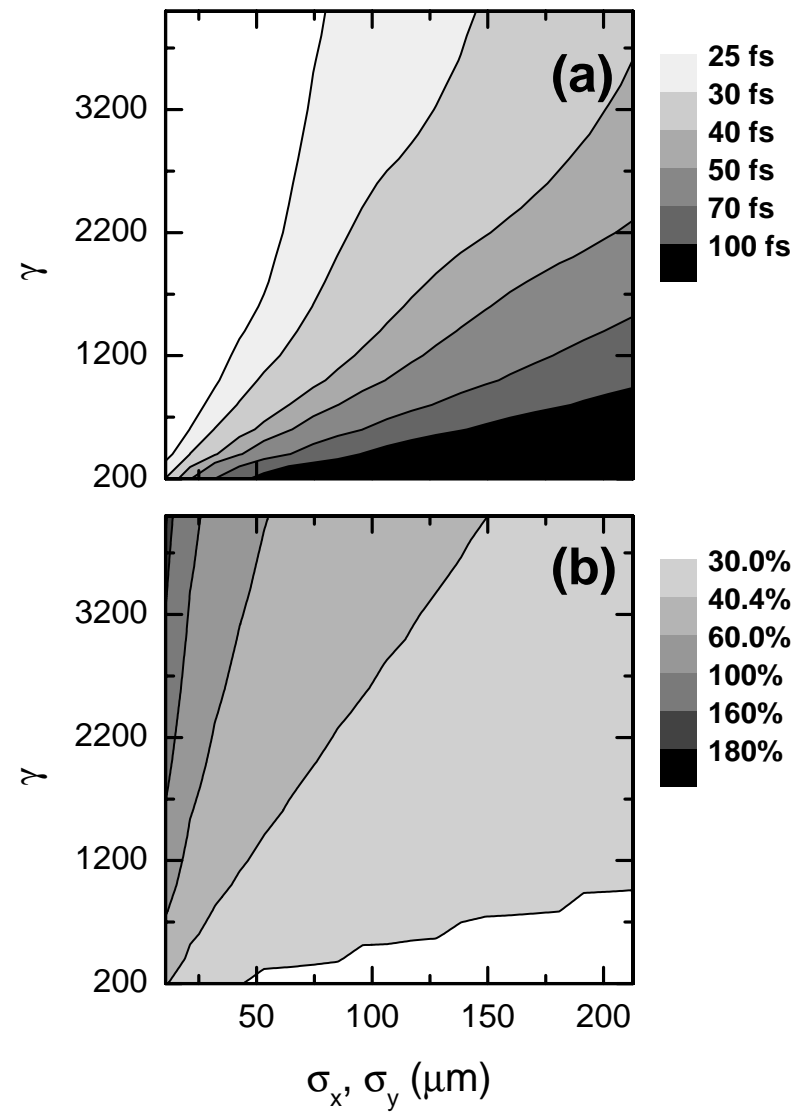

FIG. 1. Contour plots of (a) x-ray pulse FWHM duration in femtoseconds and (b) angle-integrated x-ray FWHM bandwidth $(\Delta E / E)_{\text {int }}$ as a function of transverse bunch size and energy with an rms bunch length of 0.212 ps and a normalized emittance of $10^{-5} \mathrm{mrad}$. The laser is a 20 -fs Ti:sapphire system at $800 \mathrm{~nm}$. The x-ray spectra peak at $8 \mathrm{keV}$.

of $10^{-5} \mathrm{mrad}$ is used, which is a factor of 2-3 larger than that produced from modern photoinjectors.

In the numerical examples throughout this paper we use an FWHM laser pulse duration of $20 \mathrm{fs}$; however, the SATS scheme can work with lasers having much shorter pulse duration in order to generate even faster x-ray pulses. The temporal broadening of the x-ray pulse relative to the laser pulse, defined as $\eta=\tau / \tau_{L}-1$, can be derived from Eq. (3) and used to place a limit on the maximum electron bunch transverse size (assuming a round electron bunch),

$$
\sigma_{x, y_{\max }} \approx 2\left(\eta \frac{E_{L}}{E}\right)^{1 / 2} \gamma c \tau_{L} .
$$

Here, $\phi=\left(E / E_{L}\right)^{1 / 2} / \gamma$ is used. As an example, for $\gamma=1300, E=8 \mathrm{keV}$, and $E_{L}=1.55 \mathrm{eV}$ (800 nm laser radiation), if one wishes to maintain the x-ray pulse duration within a chosen broadening factor of, for instance, $\eta=20 \%$, the maximum allowed beam size is then approximately 16 times the laser pulse length. Even for a 5 -fs FWHM pulse duration, this gives a $24-\mu \mathrm{m}$ FWHM transverse bunch size, a moderate number for high quality, well-focused electron beams. The same longitudinal or 
transverse beam size would generate 80 -fs pulses in regular Thomson scattering and synchrotron radiation schemes.

\section{Photon production and spectra}

Two disadvantages arise from small laser incidence angles. The obvious disadvantage is that the number of the scattered photons can be significantly reduced. This is due to the fact that the effective laser flux seen by the electrons becomes smaller. In the laboratory frame, the electrons (propagating at $\sim c$ ) see the photons propagating at a speed of $c(1-\cos \phi)=c \phi^{2} / 2$ along the $z$ axis, which is the only useful component of the photon flux (the transverse scattering cross section is collapsed by the Lorentz contraction effect). Considering that the interacting electrons are those underlying the laser pulse and the interaction time is $t_{c}$, the total number of scattered photons is calculated as

$$
N \approx \frac{\Sigma_{0}}{4 \pi} \frac{N_{e} N_{p}}{\sigma_{y} \sigma_{z}} \phi
$$

Here $N_{e, p}$ are numbers of electrons and photons, $\Sigma_{0}$ is the integrated scattering cross section over the acceptance angle (when integrated over all angles, it is the Thomson scattering cross section).

The second disadvantage is that the full bandwidth becomes larger as the incidence angle becomes smaller. To understand this, we first calculate the bandwidth at a particular scattering angle. This can be obtained by differentiating Eq. (1) using Gaussian energy and angular distributions for the laser electron bunches and is

$$
\frac{\Delta E}{E} \approx\left(\frac{\sigma_{\phi}^{2}+\sigma_{x^{\prime}}^{2}+\sigma_{y^{\prime}}^{2}}{\phi^{2}}+4 \sigma_{e}^{2}+\sigma_{L}^{2}\right)^{1 / 2} .
$$

Here $\sigma_{x^{\prime}, y^{\prime}}$ are the electron beam divergence, $\sigma_{e}$ is the electron bunch slice energy spread over the interaction region with the laser pulse, and $\sigma_{\phi}=\lambda_{L} / 4 \pi \sigma_{y}$ and $\sigma_{L}=$ $\lambda_{L} / 4 \pi c \tau_{L}$ are the diffraction-limited divergence and the transform-limited bandwidth of the laser pulse. To obtain the angle-integrated bandwidth, one needs to numerically convolve Eq. (5) with Eqs. (1) and (2). At incident angles comparable to or smaller than the laser divergence, spectrum broadening is dominated by the laser divergence,

$$
\left(\frac{\Delta E}{E}\right)_{\text {int }}=2 \frac{\sigma_{\phi}}{\phi}=\frac{\lambda_{L}}{2 \pi \sigma_{y} \phi} .
$$

The spectrum broadening is illustrated in Fig. 1(b), where the numerically calculated FWHM bandwidth of the angle-integrated spectra as a function of the beam energy and transverse beam size is given. The bandwidth changes from below 30\% for low beam energies and large transverse sizes to $\sim 200 \%$ at high beam energies with small transverse beam sizes, in agreement with Eq. (5a). This spectral broadening shifts the peak of the spectrum to lower energy and a larger laser incidence angle is used in the calculation to compensate this effect to maintain the peak position. For example, for an $8-\mathrm{keV}$ x-ray photon energy, when no broadening is considered, $\phi=56 \mathrm{mrad}$ for $\gamma=1270$ and $\lambda_{L}=0.8 \mu \mathrm{m}$, but with the broadening due to a beam waist of $11 \mu \mathrm{m}, \phi=62 \mathrm{mrad}$.

Summarizing Eqs. (3)-(5), the average photon flux can be estimated as

$$
F \approx \frac{\Sigma_{0}}{4 \pi} \frac{N_{p} N_{e}}{\sigma_{z}} \frac{\delta_{\mathrm{BW}}}{\lambda} \frac{1}{\gamma^{2}} f,
$$

where $\delta_{\mathrm{BW}}$ is the required bandwidth for applications, $\lambda$ is the wavelength of the scattered photons, and $f$ is the repetition rate. Here we used $\phi=\left(E / E_{L}\right)^{1 / 2} / \gamma=$ $\left(\lambda_{L} / \lambda\right)^{1 / 2} / \lambda$ in obtaining Eq. (6).

From Eq. (6), it is clear that the photon flux for a fixed x-ray photon energy decreases rapidly as the electron bunch becomes more energetic. The numerically calculated photon flux is given in Fig. 2(a) for the same condition as in Fig. 1 except that a 2-J per pulse laser energy at $6 \mathrm{~Hz}$ is used. Note that the bandwidth of the angle-integrated spectrum is used.

Obviously, for more efficient scattering or higher photon flux, larger laser incidence angles are more desirable. From Eq. (3a), for a fixed bunch size and with a relative broadening factor of $\eta=\tau / \tau_{L}-1$, the minimum electron bunch energy (corresponding to the maximum laser incidence angle) that maximizes the photon flux is

$$
\gamma_{\min } \approx \frac{\sigma_{x, y}}{2 c \tau_{L}}\left(\frac{E}{\eta E_{L}}\right)^{1 / 2}
$$

As an example, for a laser FWHM pulse length of $20 \mathrm{fs}$ at $800 \mathrm{~nm}$ and electron bunch size of $11 \mu \mathrm{m} \mathrm{rms}$, we have $\gamma_{\min }=348$, or $180 \mathrm{MeV}$ of beam energy for 24-fs $8-\mathrm{keV}$ $\mathrm{x}$-ray pulses with the laser incidence angle of about $0.2 \mathrm{rad}$. This would increase the photon flux by more than a factor of 10 when compared with the case when $\gamma=1270$ (650 MeV beam energy), with a laser incidence angle of $62 \mathrm{mrad}$.

\section{Divergence and peak spectral brightness}

However, for low-emittance electron bunches, even with fewer scattered photons and broader spectra, it can be shown that the spectral brightness (defined as photon flux per unit solid angle and area) of the x-ray burst does not necessarily degrade at small laser incidence angles. This is due to the fact that the higher beam energy enhances the Lorentz contraction effect and further collapses the divergence of the scattered photons. This divergence is a convolution of the Lorentz contraction effect of $1 / \gamma$ and the divergence of the electron bunches $\sigma_{x^{\prime}, y^{\prime}}, \varphi_{x, y} \sim$ $\left(1 / \gamma^{2}+\sigma_{x^{\prime}, y^{\prime}}^{2}\right)^{1 / 2}$. For high-quality, low-emittance electron bunches, the bunch divergence can be much smaller in most of the cases, hence the divergence of the scattered photons can be approximated by $1 / \gamma$, which decreases rapidly as electron beam energy increases.

This reduction of the divergence roughly compensates the reduced photon production and the broadened spectrum at smaller laser incidence angle. To summarize, the 
achievable peak spectral brightness of the scattered photons can be estimated as

$$
B \approx \frac{\sqrt{2} \Sigma_{0}}{16 \pi^{3}} \frac{N_{p} N_{e}}{\sigma_{x} \sigma_{y} \sigma_{z} \tau_{L}} \frac{\delta_{\mathrm{BW}}}{\lambda} .
$$

Equation (8) is valid when $1 / \gamma>\sigma_{x^{\prime}, y^{\prime}}$. It should also be mentioned that, due to the scattering geometry, the source sizes in the $x$ and $y$ directions are calculated differently. In the $y$ direction, it is determined by overlapping two Gaussian profiles with the same width; hence the profile is narrowed and is $\sigma_{y} / \sqrt{2}$. In the $x$ dimension, the laser pulse "scans" the electron bunch; hence the bunch size determines the source size, which is $\sim \sigma_{x}$.

The numerically calculated peak spectral brightness is given in Fig. 2(b). One sees that there is clearly an optimal beam energy that maximizes the spectral brightness, which occurs when $1 / \gamma<\sigma_{x^{\prime}, y^{\prime}}$ and the laser divergence $2 \sigma_{\phi} / \phi$ starts to dominate the angle-integrated bandwidth $(\Delta E / E)_{\text {int }}$.

Figure 2 actually shows SATS performance achievable with current laser and accelerator technologies. With an
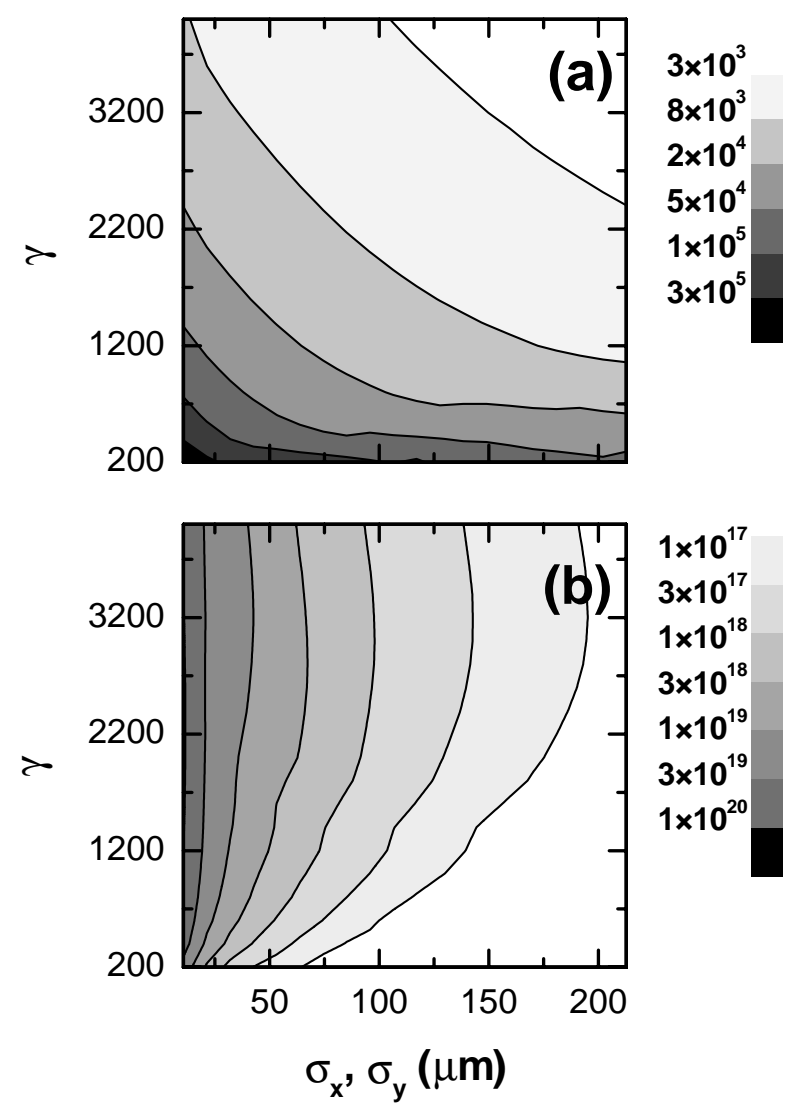

FIG. 2. Contour plots of (a) the average photon flux (photons $\mathrm{s}^{-1}$ per $0.1 \%$ bandwidth) and (b) the spectral brightness (photons s $\mathrm{mm}^{-2} \mathrm{mrad}^{-2}$ per $0.1 \%$ bandwidth) as a function of transverse bunch size and beam energy. The rms bunch length is $0.212 \mathrm{ps}$, and the normalized emittance is $10^{-5} \mathrm{mrad}$. The laser is a 20-fs, 2-J Ti:sapphire system at $800 \mathrm{~nm}$ at $6 \mathrm{~Hz}$. The $\mathrm{x}$-ray spectra peak at $8 \mathrm{keV}$. A repetition rate of $6 \mathrm{~Hz}$ is used when calculating the average photon flux. electron bunch charge of $1 \mathrm{nC}$, laser pulse energy of $2 \mathrm{~J}$ in a duration of $20 \mathrm{fs}$ [18], the peak brightness obtainable is about $10^{20}$ photons $\mathrm{s}^{-1} \mathrm{~mm}^{-2} \mathrm{mrad}^{-2}$ per $0.1 \% \mathrm{BW}$ at $8 \mathrm{keV}$. The electron beam has a normalized emittance of $10^{-5} \mathrm{mrad}$ and beam energy of $650 \mathrm{MeV}(\gamma \sim 1270)$. This is a flux of $\sim 10^{5}$ photons per second per $0.1 \%$ bandwidth in a duration of $20 \mathrm{fs}$ and a cone of $3 \mathrm{mrad}$. The peak brightness is close to that of the third-generation synchrotron light sources.

\section{E. Tunability}

It is trivial to see that, by adjusting the laser incidence angle, the peak of the x-ray spectra can be easily adjusted. In Fig. 3, for x-ray spectra peaks of up to $40 \mathrm{keV}$, there is no significant change in the properties of the scattered photons. In Fig. 3(a), the spectra with peak photon energy from 8 to $40 \mathrm{keV}$ are given, and Fig. 3(b) gives the pulse duration and peak spectral brightness as a function of the spectrum peak position. Clearly, the duration is almost constant at 20 fs as the spectrum peak shifts from 8 to $40 \mathrm{keV}$. The only observed change is the increase in the peak spectral brightness when the spectrum shifts to higher peak photon energy due to the larger laser incidence angle.

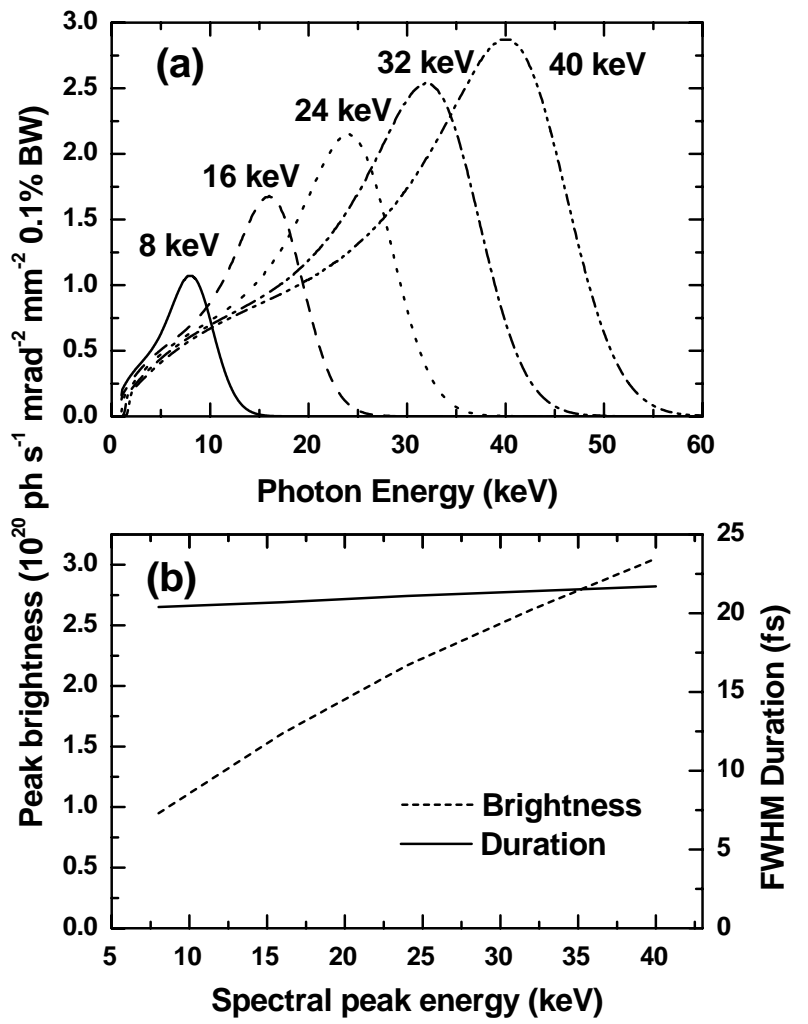

FIG. 3. (a) Spectra with peak positions at 8 to $40 \mathrm{keV}$ and (b) peak brightness (dotted line) and pulse duration (solid line) as a function of the spectrum peak position. The bunch energy is $650 \mathrm{MeV}$ with rms bunch length of $0.212 \mathrm{ps}$, and the normalized emittance is $10^{-5} \mathrm{mrad}$. The laser is a $20-\mathrm{fs}, 2-\mathrm{J}$, Ti:sapphire system at $800 \mathrm{~nm}$. Shifting the spectrum peak is accomplished by changing the laser incidence angle. 


\section{BEHAVIOR AT HIGH LASER INTENSITY}

\section{A. Formulas}

While the above discussion is valid in the linear regime, to maximize spectral brightness of the source, high laser intensity is needed. For example, in the calculations in Figs. 2 and 3, for the tightest focus case of $w_{0}=11 \mu \mathrm{m}$, we have for a 2-J, 20-fs laser pulse an intensity of $I \sim 10^{19} \mathrm{~W} / \mathrm{cm}^{2}$; the normalized laser strength parameter is $a=8.6 \times 10^{-9} \lambda_{L}[\mu \mathrm{m}]\left(I\left[\mathrm{~W} \mathrm{~cm}^{-2}\right]\right) \sim 3$. In this regime, the electron motion in the laser field becomes highly nonlinear, and effects such as ponderomotive scattering $[19,20]$ and harmonic generation $[15,16]$ become important. While the generation of harmonics gives a larger tuning range, the ponderomotive scattering has the potential of deflecting the electrons from the laser focus before they can scatter the photons.

Analyses of electron dynamics $[21,22]$ and resultant radiation $[15,16]$ in laser fields of relativistic intensities have been presented by several authors. Here we present a full time- and space-dependent calculation of the electron dynamics using a laser modeled by the angular-spectrum method [19]. For a wave polarized in the $x$ direction it gives

$$
\begin{aligned}
& E_{x}=\frac{E_{0}}{4 \varepsilon^{2}}\left(I_{1}+\frac{x^{2}+y^{2}}{k_{0} r^{3}} I_{2}+\frac{y^{2}}{r^{2}} I_{3}\right), \\
& E_{y}=\frac{E_{0}}{4 \varepsilon^{2}} \frac{x y}{k_{0} r^{3}}\left(k_{0} r I_{3}-2 I_{2}\right), \\
& E_{z}=\frac{E_{0}}{4 \varepsilon^{2}} \frac{x}{r} I_{4}, \\
& B_{x}=\frac{E_{y}}{c}, \\
& B_{y}=\frac{E_{0}}{4 c \varepsilon^{2}}\left(I_{1}+\frac{y^{2}-x^{2}}{k_{0} r^{3}} I_{2}+\frac{x^{2}}{r^{2}} I_{3}\right), \\
& B_{z}=\frac{E_{0}}{4 c \varepsilon^{2}} \frac{y}{r} I_{4},
\end{aligned}
$$

where

$$
\begin{gathered}
I_{1}=\int_{0}^{1} e^{-b^{2} / 4 \varepsilon^{2}}\left(1+\sqrt{1-b^{2}}\right) \sin \left(\phi_{b}\right) \\
\quad \times J_{0}\left(k_{0} r b\right) b d b, \\
I_{2}=\int_{0}^{1} e^{-b^{2} / 4 \varepsilon^{2}} \frac{\sin \left(\phi_{b}\right)}{\sqrt{1-b^{2}}} J_{1}\left(k_{0} r b\right) b^{2} d b, \\
I_{3}=\int_{0}^{1} e^{-b^{2} / 4 \varepsilon^{2}} \frac{\sin \left(\phi_{b}\right)}{\sqrt{1-b^{2}}} J_{0}\left(k_{0} r b\right) b^{3} d b, \\
I_{4}=\int_{0}^{1} e^{-b^{2} / 4 \varepsilon^{2}}\left(1+\frac{1}{\sqrt{1-b^{2}}}\right) \cos \left(\phi_{b}\right) \\
\quad \times J_{1}\left(k_{0} r b\right) b^{2} d b,
\end{gathered}
$$

$$
\phi_{b}=\omega_{0} t-k_{0} z \sqrt{1-b^{2}}+\phi_{0} .
$$

Here $k_{0}$ is the wave vector and $\varepsilon=1 / k_{0} w_{0}$ with $w_{0}$ as the laser beam waist. The radiation power is calculated using the Lienard result [21]

$$
P=\frac{2}{3} \frac{e^{2}}{c} \gamma^{6}\left[\dot{\boldsymbol{\beta}}^{2}-(\boldsymbol{\beta} \times \dot{\boldsymbol{\beta}})^{2}\right],
$$

and the radiation spectrum is calculated using the LienardWiechert potentials [21]

$$
\frac{d^{2} I}{d \omega d \Omega}=\frac{e^{2} \omega^{2}}{4 \pi^{2} c}\left|\int \mathbf{n} \times(\mathbf{n} \times \boldsymbol{\beta}) e^{i \omega(t-\mathbf{n} \cdot \mathbf{r} / c)} d t\right|^{2},
$$

where $\mathbf{n}=\mathbf{e}_{x} \sin \theta \cos \varphi+\mathbf{e}_{y} \sin \theta \sin \varphi+\mathbf{e}_{z} \cos \theta$ and $\varphi$ is the azimuthal angle. An 800-nm, 20-fs FWHM Gauss pulse with a beam waist of $10.5 \mu \mathrm{m}$ is used in the calculation; the initial phase $\phi_{0}=0$ unless indicated.

\section{B. Electron dynamics}

Figure 4 shows the trajectories and the scattering angle of an electron with $\gamma=1270$ as a function of $a_{0}$ at a laser interaction angle of $\phi=62 \mathrm{mrad}\left(3.55^{\circ}\right)$. The laser pulse is polarized in the incidence plane of the laser
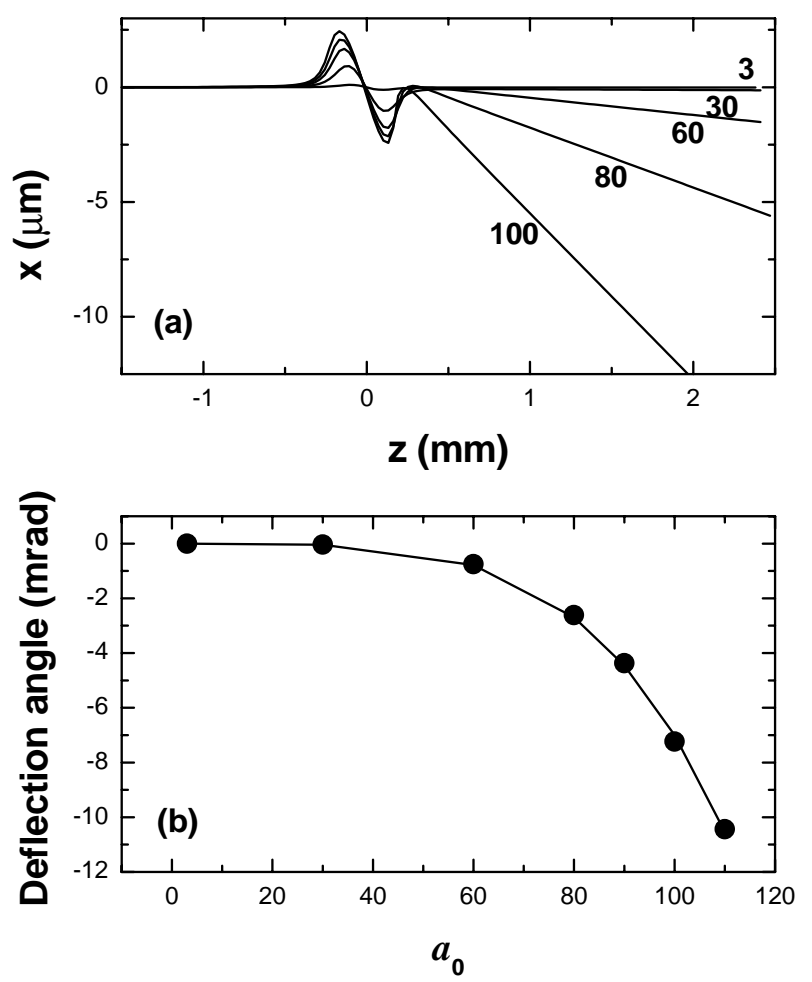

FIG. 4. (a) The trajectories of an electron with $\gamma=1270$ as a function of $a_{0}$, at an interaction angle of $\phi=62 \mathrm{mrad}$ $\left(3.55^{\circ}\right)$. The laser pulse has a $\tau_{\mathrm{FWHM}}=20 \mathrm{fs}$ at a wavelength of $\lambda_{L}=0.8 \mu \mathrm{m}$ with the polarization in the incidence plane of the laser ( $p$ polarization). In the calculation, the electron and the laser meet at $x, y, z=0$ at $t=0$. (b) Electron scattering angle after the interaction as a function of $a_{0}$. 
( $p$ polarization). In the calculation, the electron and the laser meet at $x, y, z=0$ at $t=0$. This is the optimized case from Sec. II for 8-keV x-ray generation.

Clearly, in Fig. 4, the electron is scattered and changes its propagation direction after the interaction. However, we note that the scattering is an order of magnitude lower than expected from the ponderomotive scattering effect. The ponderomotive force is [19]

$$
\frac{d \mathbf{p}}{d t} \approx-\frac{m c^{2}}{\gamma} \nabla a^{2}
$$

With the laser focus size of $\sigma_{x}$, one has $\nabla_{\perp} a^{2} \approx a^{2} / \sigma_{x}$ and an interaction time of $t_{c} \approx \sigma_{x} / c \phi$. The maximum change in the transverse momentum is approximately $\delta p_{\perp} \approx m c a^{2} / \gamma \phi$, therefore the change in the propagation direction is $d \phi \sim \delta p_{\perp} / p \approx-(a / \gamma)^{2} / \phi$. For the condition $(\gamma=1270, \phi=62 \mathrm{mrad})$ used in the simulation, this gives $d \phi \approx 10^{-5} a^{2}$, while fitting the data in Fig. 4(b) gives $d \phi \approx-1.8 \times 10^{-11} a^{4.3}$, a smaller effect but a stronger dependence on the laser intensity. Adding to the evidence is the dependencies of the scattering angle upon the initial phase and the polarization of the laser pulse, which should have no impact on the ponderomotive motion of the electron.

This deviation from the ponderomotive effect is not a surprise because the ponderomotive force is more an averaging effect due to the laser intensity gradient, while in our case the electron experiences only a few oscillations, voiding the averaging effect. In fact, the field and its time dependence become more critical.

Clearly, from Fig. 4(b), it is evident that, for the intensity regime we are interested in, the laser scattering effect is not important at all. We note that the calculation also shows significant energy modulation of the electron during the interaction. For a modest value of $a=3$ the energy modulation of up to 20 times the expected value of $a^{2} / 2$ is observed for the case of $p$ polarization, while only negligible energy modulation is observed for the $s$-polarization case (polarization perpendicular to the laser incidence plane).

\section{Radiation}

The spectra calculated using Eq. (12) at different laser strengths for $\theta=0$ is given in Figs. 5(a) -5 (c) for $a_{0}=$ $0.1,1$, and 2 , respectively. We notice several effects. The first is a strong blueshift of the spectrum peak for the $a_{0}=0.1$ case. This blueshift is found to be dependent on the size of the beam waist: the stronger the laser focus, the larger the blueshift. This blueshift can be explained by the linear theory in Sec. II as the result of the dependence of the scattering efficiency and the scattered photon energy on the laser photon incidence angle: the larger the angle the higher the x-ray photon energy and higher scattering efficiency [Eqs. (1) and (4)]. For comparison, the spectrum for a weakly focused laser $\left(w_{0}=100 \mu \mathrm{m}\right)$, with all other conditions the same, is given in Fig. 5(a) as the dashed

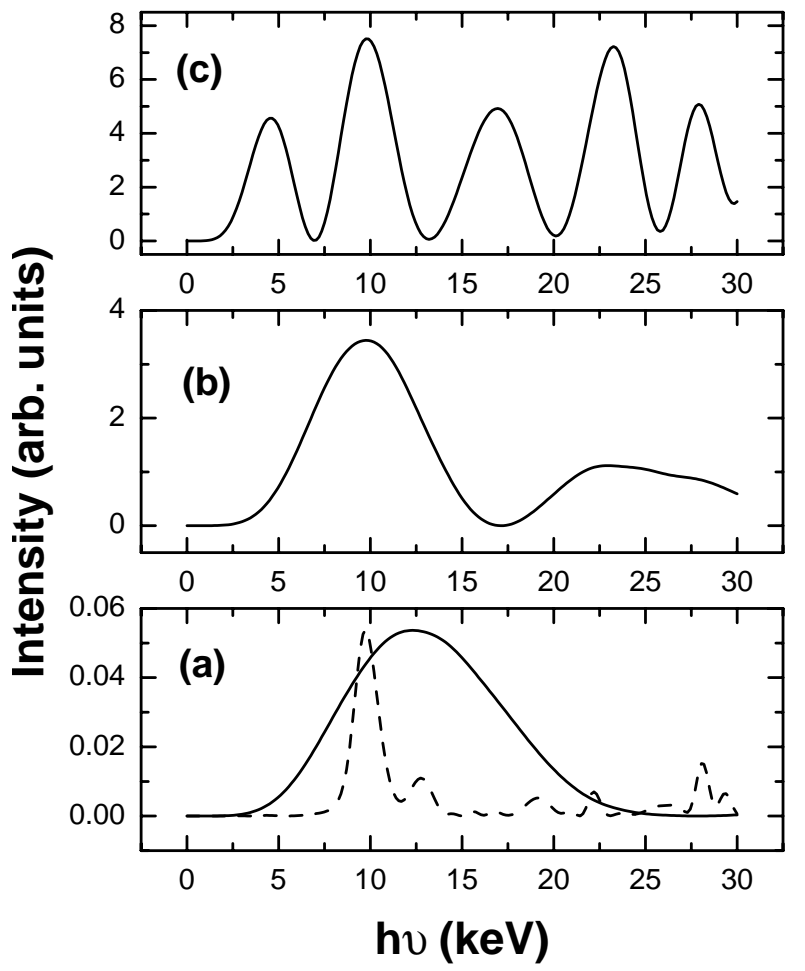

FIG. 5. Spectra for (a) $a_{0}=0.1$, (b) $a_{0}=1$, and (c) $a_{0}=2$. A spectrum for a weakly focused $\left(w_{0}=100 \mu \mathrm{m}\right)$ laser beam is also given for comparison in (a) (dashed line).

line. Here the spectrum peaks at the expected position of $9600 \mathrm{eV}$ as predicted by linear theory [Eq. (1)]. The much broader spectrum for tighter spectrum is obviously due to the laser divergence, which is automatically included in the angular spectrum representation of the laser field in Eq. (9). The spectrum is very similar to those in Fig. 3.

The second effect is the generation of harmonics and the redshift of the harmonic spectra as predicted by theory [15]. For $\gamma \gg 1$ and $\theta \ll 1$,

$$
\omega_{n}=n \frac{2 \gamma^{2} \omega_{0}}{1+a^{2} / 2+\gamma^{2} \theta^{2}}(1-\cos \phi) .
$$

Here $n$ is the harmonic number. We mention that the theory is valid strictly for a plane wave laser field at constant intensity, hence it cannot be used to describe the complex features due to temporal and spatial dependence of the laser field.

As can be seen in Fig. 5, the temporal and spatial dependence of the laser field causes significant spectrum broadening, hence, at high intensity, the harmonics of different orders overlap with each other. Depending on the emission phase of the harmonics, they can interfere with each other and make the spectrum extremely complicated, an effect that needs further investigation.

Finally, the harmonics (including the fundamental) radiation shows saturation as the laser strength parameter increases beyond 1. This is shown in Fig. 6(a), where the peak intensity of the fundamental is depicted as a function 

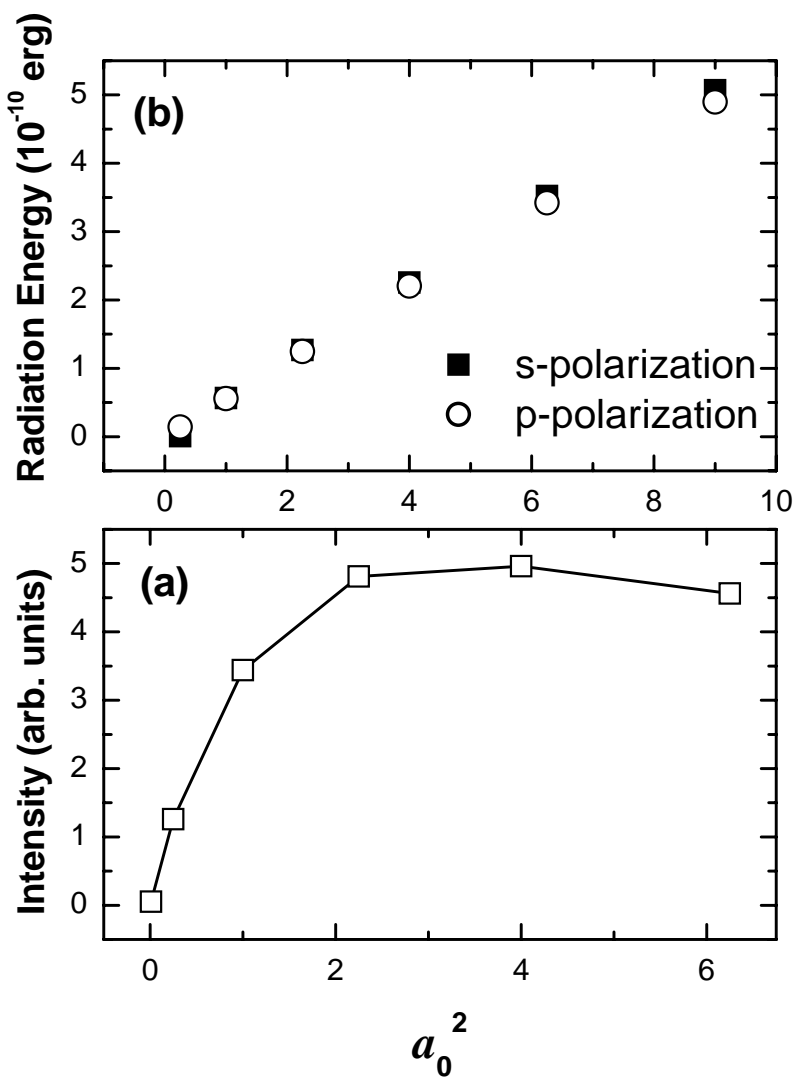

FIG. 6. (a) The peak intensity of the fundamental and (b) the total radiated energy as a function of the laser intensity $\left(a_{0}^{2}\right)$.

of the laser intensity $\left(a_{0}^{2}\right)$. One can see that the intensity increases quickly for $a_{0}<1$, and starts to saturate at $a_{0}=1.5$ to 2 . This is very important for practical operation of the SATS source: the saturation is limiting the obtainable spectral brightness. This is quite apart from the ponderomotive scattering effect suspected previously [22].

The saturation of the fundamental is accompanied by the appearance of new harmonics, as seen in Figs. 5(b) and $5(\mathrm{c})$. So while the fundamental and harmonics saturate, the total power of the radiation increases due to the appearance of new harmonics. The total radiation energy, by integrating Eq. (11) over time, is given in Fig. 6(b), showing a linear dependence on the laser intensity or $a_{0}^{2}$, in agreement with previous analyses $[15,23]$. The generation of harmonics opens up the tunable range of the SATS source without reducing the spectral brightness. Meanwhile, due to the generation of high harmonics, an estimate of the practical brightness limit is complicated by the emerging higher harmonics and the overlap between them.

\section{DISCUSSION}

\section{A. Linac electron source and storage ring electron source}

From Fig. 2 and Eqs. (6) and (8), we clearly see the importance of the phase-space density of the electron bunch for high brightness. Generally, the denser the bunch in phase space, the higher the brightness, thus highlighting the importance of using a linear accelerator for this application.

A storage ring is a circulating accelerator, where the final beam quality is determined by the intrinsic equilibrium between the synchrotron radiation dumping and the quantum excitation effect. In comparison, a linear accelerator has two major advantages. The first is that it is a one-pass machine, making it possible to preserve the low emittance of electron bunches generated using modern photocathode guns. The second advantage of a linac is the freedom of using bunch compressors to shorten the bunch length to well below 1 ps. At the Advanced Photon Source (APS) injection linac [24,25], for example, normalized rms emittance smaller than $10^{-5} \mathrm{mrad}$ and rms bunch length of $\sim 0.2 \mathrm{ps}$ have been routinely achieved. With an energy spread of $0.1 \%$, particle-tracking simulation shows it is possible to achieve an $11-\mu \mathrm{m}$ transverse beam size at $650 \mathrm{MeV}$ with modest effort. In a storage ring, the requirement for stable betatron and synchrotron oscillations largely limit the flexibility of manipulating the stored bunches.

However, a storage ring has the advantage of high repetition rate up to $\mathrm{MHz}$. In this case, with high repetition rate laser systems, it is possible to obtain higher average spectral brightness, especially when the filling pattern in the storage ring is such that multiple interaction of the laser pulse can be arranged.

\section{B. Comparison with other sources}

Our calculation is summarized in Table I. Table I also lists the performance of other subpicosecond $\mathrm{keV} x$-ray sources, namely, the right-angle and head-on Thomson scattering, the bunch-sliced synchrotron radiation, and that of laser plasma sources.

The laser-plasma $\mathrm{x}$-ray sources are compact table-top sources based on the $\mathrm{K} \alpha$ atomic radiation from the target materials excited by hot electrons accelerated by the laser field [10]. Hence, even the fundamental time is the rate of the atomic transition of a few femtoseconds; the practical need of generating a low density plasma for hot electron production and the finite penetration depth of the hot electrons in the target material makes it difficult to shorten the $\mathrm{x}$-ray pulse duration to below $100 \mathrm{fs}$. The radiation is spread in $4 \pi$ solid angles.

The bunch sliced synchrotron radiation [11] can deliver very high average photon flux due to the high repetition rate. Note that the x-ray pulse repetition rate is not determined by the repetition rate of the electron bunches but by the radiation damping rate and the number of electron bunches in the storage ring (see Ref. [11] for detail). However, the technique relies on the dispersion of the electron bunch in the circular accelerators to separate the sliced bunch (with modified bunch energy) from the mother bunch. During this process, the bunch is stretched 
TABLE I. Summary of the proposed APS SATS X-ray source.

\begin{tabular}{|c|c|c|c|c|c|}
\hline & APS linac ${ }^{a}$ & ALS $90 \mathrm{TS}^{\mathrm{b}}$ & ALS slicing ${ }^{c}$ & BNL $180 \mathrm{TS}^{\mathrm{d}}$ & Laser plasma $^{\mathrm{e}}$ \\
\hline Wavelength $(\AA)$ & $1.5-0.3$ & 0.4 & 6 & 1.8 & $1-10$ \\
\hline Repetition rate $(\mathrm{Hz})$ & 6 & 100 & $10^{5}$ & & 10 \\
\hline Pulse length (fs) & 20 & 300 & $\sim 100$ & 3500 & $\sim 300$ \\
\hline Average photon flux ${ }^{\mathrm{e}}$ & $10^{6}$ & $10^{5}$ & $10^{7}$ & $10^{5}$ & $10^{9}$ \\
\hline Divergence (mrad) & 3 & 10 & 0.6 & & $4 \pi$ \\
\hline Bandwidth $^{f}$ & $67 \%-200 \%$ & $80 \%$ & & $28 \%$ & $<0.1 \%$ \\
\hline Peak brightness ${ }^{g}$ & $\sim 10^{20}$ & $3 \times 10^{15}$ & $\sim 10^{19}$ & $10^{16}$ & $\sim 10^{18}$ \\
\hline
\end{tabular}

aperating with a 6-Hz, 20-fs, 2-J, 800-nm laser at $650 \mathrm{MeV}$ beam energy may need a factor of 2-3 adjustment for saturation effect. ${ }^{b}$ Reference [6], perspective value.

${ }^{\mathrm{c}}$ Reference [11], perspective value.

${ }^{\mathrm{d}}$ Reference [8], experimental data.

eReference [10], experimental data.

${ }^{\mathrm{f}}$ In photons $\mathrm{s}^{-1}$ per $0.1 \%$ bandwidth.

gIn photons $\mathrm{s}^{-1} \mathrm{~mm}^{-2} \mathrm{mrad}^{-2}$ per $0.1 \% \mathrm{BW}$.

due to its large energy spread and the current proposed parameter ranges from $100-200$ fs and the ability to access shorter pulse duration is limited.

For the three Thomson scattering geometries listed in Table I, the duration and photon production of the $\mathrm{x}$-ray pulses have different dependences upon the laser electron beam parameters. For the SATS, the advantage is that the pulse duration is solely determined by the interaction laser pulse [Eq. (3)] and hence is capable to push the $\mathrm{x}$-ray pulse duration to what is available so far only in optical laser systems of less than $20 \mathrm{fs}$. With given electron parameters, the photon production is limited by the saturation of the scattering due to the limited interaction cycles in the geometry as discussed in Sec. IIIC. For $90^{\circ}$ scattering [5,6], the pulse durations are determined both by the transverse size of the electron bunches and the laser pulse duration. The photon production is higher but still limited by the saturation for given electron parameters. For the head-on scattering, the duration of the $\mathrm{x}$-ray pulses is solely determined by the electron bunch length, but the photon production efficiency is no longer limited because one can use very long laser pulse [8] or even standing laser waves to implement as many interaction cycles as practically possible, hence very high photon production is possible. The challenge is then to develop ultrashort electron bunches with bunch length of sub-100 fs $[26,27]$, similar to those proposed ultrafast $\mathrm{x}$-ray facilities using undulator radiations [28-30], including $\mathrm{x}$-ray free-electron lasers. However, the current record bunch length is $50 \mathrm{fs}$ rms with $70 \mathrm{pC}$ of charge [27].

Clearly, based on already mature laser and accelerator technologies, SATS is an immediate and unique solution for obtaining bright sub-100-fs to 20-fs x-ray pulses for many existing linacs. In fact, SATS stands out in Table I because of two distinctive characteristics: short pulse duration of well below 100 fs and higher peak spectral brightness beyond $10^{20}$ photons $\mathrm{s}^{-2} \mathrm{~mm}^{-2} \mathrm{mrad}^{-2}$ per $0.1 \%$ bandwidth.

As has been discussed above, the limitation of the scalability of the SATS scheme is due to the saturation of the harmonic radiation. In this case, the highest laser intensity required for the best spectral brightness is on the order of $a=1-3$, or $I=10^{18}-10^{19} \mathrm{w} \mathrm{cm}^{-2}$. Because of this effect, the SATS brightness in Table I may need to be adjusted by a factor of $2-3$. An accurate adjustment needs a full evaluation of all the electrons involved in the interaction. Note that, while higher laser intensity does not add to the spectral brightness, the generation of harmonics gives advantages to lower energy machines that use SATS for ultrashort pulse x-ray sources of the desired x-ray photon energy.

The major challenge for implementing the SATS is to synchronize the electron bunch and the laser pulse accurately to minimize the brightness fluctuation. Analysis shows that the average spectral brightness will be reduced by a factor of $\left[1+\left(c t_{j} / \sigma_{z}\right)^{2}\right]^{-1 / 2}$ for a jitter of $t_{j}$ between the laser pulse and the electron bunch. With a bunch length of $0.2 \mathrm{ps}$, and a 1-ps jitter (measured between the current linac and the photocathode gun drive laser at the APS injection linac), a 5-times reduction on the average brightness is expected. Lengthening the bunch length to the jitter size can give a better shot-to-shot fluctuation but lower nominal peak brightness. However, because the same laser pulse used for x-ray production can also be used to pump the sample under study, very accurate timing for a pump-probe experiment can be expected. For other x-ray sources not originated from a laser pulse, pinching down the synchronization between the laser pulse and the x-ray pulse remains a challenge for accurate pump-probe experiments.

\section{CONCLUSION}

In summary, we propose the use of small-angle Thomson scattering for sub-100-fs keV x-ray pulse generation. As an example, we show that, by using the high-quality electron bunches in the APS injection linac, 8-40-keV $\mathrm{x}$-ray pulses can be generated with a peak spectral brightness of $10^{20}$ phontons $\mathrm{s}^{-1} \mathrm{~mm}^{-2} \mathrm{mrad}^{-2}$ per $0.1 \%$ bandwidth and with pulse durations of $\sim 20 \mathrm{fs}$. The technique 
can also be used for sub-10-fs x-ray pulse generation. The scheme can be optimized either for higher photon flux or for higher spectral brightness by changing the laser incidence angle and the electron bunch energy to best fit the experiment's requirement. The preliminary calculation of the electron dynamics at high laser intensity verified the spectral property in the linear analysis and indicates other interesting features.

\section{ACKNOWLEDGMENTS}

The authors thank B. Hafizi of Navy Research Laboratory and P. Mora of Ecole Ploytechnique for helpful discussion on the effect of the ponderomotive scattering. This work is supported by the U.S. Department of Energy, Office of Basic Energy Sciences, under Contract No. W-31-109-ENG-38.

[1] F. R. Arutyumian and V. A. Tumanian, Phys. Lett. 4, 176 (1963).

[2] R. H. Milburn, Phys. Rev. Lett. 10, 75 (1963).

[3] J. S. Fraser, R. L. Sheffield, and E. R. Gray, Nucl. Instrum. Methods Phys. Res., Sect. A 250, 71 (1986).

[4] S. Backus, C. G. Durfee III, M. M. Murnane, and H. C. Kapteyn, Rev. Sci. Instrum. 69, 1207 (1998), and references therein.

[5] K.-J. Kim, S. Chattopadhyay, C. V. Shank, Nucl. Instrum. Methods. Phys. Res., Sect. A 341, 351 (1994).

[6] R. W. Schoenlein, W. P. Leemans, A. H. Chin, P. Volfbeyn, T. E. Glover, P. Balling, M. Zolotorev, K.-J. Kim, S. Chattopadhyay, and C. V. Shank, Science 274, 236 (1996).

[7] F. Sakai, A. Endo, S. Ito, K. Takasago, Y. Okada, T. Yanagida, J. Yang, and M. Yorozu, in Proceedings of the Particle Accelerator Conference, Chicago, 2001, http:// accelconf.web.cern.ch/AccelConf/p01/INDEX.HTM, p. 2695.

[8] I. V. Pogorelsky, I. Ben-Zvi, T. Hirose, S. Kashiwagi, V. Yakimenko, K. Kusche, P. Siddons, J. Skaritka, T. Kumita, A. Tsunemi, T. Omori, J. Urakawa, M. Washio, K. Yokoya, T. Okugi, Y. Liu, P. He, and D. Cline, Phys. Rev. ST Accel. Beams 3, 090702 (2000).

[9] D. A. Reis, M. F. DeComp, P. H. Bucksbaum, R. Clarke, E. Dufresne, M. Herltein, R. Merlin, R. Falcone, H. Kapteyn, M. M. Murnane, J. Larsson, Th. Missala, and J. S. Wark, Phys. Rev. Lett. 86, 3072 (2001), and references therein.

[10] A. Rousse, C. Rischel, and J.-C. Gauthier, Rev. Mod. Phys. 73, 17 (2001), and references therein.

[11] R. W. Schoenlein, S. Chattopadhyay, H. H. W. Chong, T. E. Glover, P. A. Heimann, C. V. Shank, A. Zholents, and M. Zolotorev, Science 287, 2237 (2000).
[12] A. H. Zewail, J. Phys. Chem. 104, 5660 (2000), and references therein.

[13] C. B. Schroeder, C. Pellegrini, S. Reiche, J. Arthur, and P. Emma, in Proceedings of the Particle Accelerator Conference, Chicago, 2001 (Ref. [7]), p. 2757.

[14] W. Brefeld, B. Faatz, J. Feldhaus, M. Koerfer, T. Moeller, J. Pflueger, J. Rossbach, E. L. Saldin, E. A. Schneidmiler, S. Schreiber, J. Krzywinski, and M. V. Yurkov, in Proceedings of the 23rd International Free Electron Conference, Darmstadt, Germany, 2001 (to be published).

[15] S. K. Ride, E. Esarey, and M. Baine, Phys. Rev. E 52, 5425 (1995), and references therein.

[16] Y. I. Salamin and F. H. M. Faisal, Phys. Rev. A 54, 4383 (1996), and references therein.

[17] V.B. Berrestetskii, E. M. Lifshitz, and L. P. Pitaevskii, Quantum Electrodynamics (Pergamon, New York, 1980), 2nd ed., Chap. X.

[18] K. Yamakawa, M. Aoyama, S. Matsuoka, T. Kase, Y. Akahane, and H. Takuma, Opt. Lett. 23, 1468-1470 (1998).

[19] B. Quesnel and P. Mora, Phys. Rev. E 58, 3719 (1998).

[20] F. H. Hartemann, S. N. Fochs, G.P. Le Sage, N. C. Luhmann, Jr., J. G. Woodworth, M. D. Perry, Y. J. Chen, and A. K. Kerman, Phys. Rev. E 51, 4833 (1995).

[21] J.D. Jackson, Classical Electrodynamics (Wiley, New York, 1976).

[22] Y. Li, Z. Huang, M. Borland, and S. V. Milton, Proc. SPIE Int. Soc. Opt. Eng. (to be published).

[23] Y. Ueshima, Y. Kishimoto, A. Sakai, and T. Tajima, Laser Part. Beams 17, 45 (1999).

[24] G. Travish, S. Biedron, M. Borland, M. Hahne, K. Harkay, J. Lewellen, A. Lumpkin, S. Milton, and N. Sereno, in Proceedings of the 20th International Linac Conference, Stanford, CA, edited by A. Chao (SLAC Report No. SLAC-R-561 2000, p. 899).

[25] M. Borland, J. Lewellen, and S. Milton, in Proceedings of the 20th International Linac Conference, edited by A. Chao (Ref. [24]), p. 863.

[26] M. Borland, in Proceedings of the Particle Accelerator Conference, Chicago, 2001 (Ref. [7]), p. 2209.

[27] P. Kung, H. Lihn, and H. Wiedemann, Phys. Rev. Lett. 73, 967 (1994).

[28] M. Tigner, I. V. Bazarov, D. H. Bilderback, S. M. Gruner, H. Padamsee, R. Talman, G. A. Krafft, L. Merminga, and C. K. Sinclair, in Proceedings of the Particle Accelerator Conference, Chicago, 2001 (Ref. [7]), p. 230.

[29] V. N. Litvinenko, S. F. Mikhailov, O. A. Shevchenko, and Y. $\mathrm{Wu}$, in Proceedings of the Particle Accelerator Conference, Chicago, 2001 (Ref. [7]), p. 2614.

[30] A. Zholents, J. N. Corlett, S. De Santis, N. Hartman, P. Heimann, R. Lafever, D. Li, H. Padmore, R. A. Rimmer, K. E. Robinson, R. Schoenlein, J. Tanabe, S. Wang, and D. Kairan, in Proceedings of the Particle Accelerator Conference, Chicago, 2001 (Ref. [7]), p. 2635. 